\title{
Intracranial venous thrombosis
}

\author{
M. PARSONS \\ M.A., M.R.C.P. \\ Senior Registrar, \\ Department of Neurology, University College Hospital, London, W.C.1
}

\begin{abstract}
Although cerebral arterial disease has been studied intensively in recent years, little attention has been paid to cerebral venous thrombosis. This condition may be encountered at any age and in any department, often in the guise of a bizarre neurological disorder, and is of considerable importance among children and young adults. Because it is so frequently misdiagnosed, and because there is such uncertainty about treatment, especially where anticoagulants are concerned, there seemed to be scope for a review in which the syndromes of cavernous sinus thrombosis, sagittal sinus and cortical venous thrombosis, lateral sinus thrombosis and thrombosis of the vein of Galen were considered in turn. Despite the tendency for one syndrome to merge into another, this is a convenient classification, but variations in each clinical picture will be encountered due to the presence or absence of infection, the speed of thrombosis, the potentialities of the collateral circulation and thromboembolic complications.
\end{abstract}

\section{Cavernous sinus thrombosis}

\section{Clinical presentation and investigations}

Cavernous sinus thrombosis, a disease of children and young adults, usually follows a staphylococcal infection of the face, mouth, sinuses, pharynx or ear. Patients present with a short history of fever, headache, facial swelling, diplopia and paraesthesiae in the trigeminal territory, and are found to have orbital oedema, chemosis, exophthalmos, external and, later, internal ophthalmoplegia. They deteriorate rapidly, developing bilateral eye signs, often followed by fits, coma, a hemiplegia and embolic complications. The blood culture is nearly always positive, but organisms are rarely grown from the cerebrospinal fluid (CSF) despite the presence of a pleocytosis in the majority (Taylor, 1957). Angiographic studies may show non-filling of the sinus on late films (Clune, 1963) but are rarely required, for this condition is diagnosed on clinical grounds. Occasional cases of aseptic cavernous sinus thrombosis due to facial trauma, sinus puncture, tumour growth, or maras- mus, and a subacute form (Barraquer Bordas, Bachs \& Barraquer-Moner, 1955) have been described.

\section{Treatment}

The key to successful treatment is the early recognition that this is a potentially lethal, rapidly progressive infection due to a virulent organism. Large doses of two or three of the most powerful broad spectrum antibiotics currently available should be given at once, without waiting for the results of sensitivity tests. Suitable treatment must be maintained for a period of 2 or 3 months, as the literature contains many reports of patients who survived the acute illness, to relapse or die later of cerebral or metastatic abscesses when antibiotics were stopped prematurely.

In addition to the administration of antibiotics, a causative ear or sinus infection may need surgical attention, and most patients will need sedatives and analgesics, with anticonvulsants if fits or a hemiplegia suggest cortical damage. About the use of anticoagulants there is still uncertainty. Protagonists argue that the responsible organism produces a coagulase, and forms a clot which acts as a nutrient medium, a source of emboli and a barrier against antibiotics. Opponents argue that anticoagulants are liable to aggravate haemorrhagic lesions in the globe and the brain, that the clot confines the infection, and that anticoagulants are useless once it has formed. No individual could hope to resolve the problem on the basis of personal experience of this rare condition, and one is forced to the unsatisfactory alternative of reviewing the series of isolated case reports in the English literature since anticoagulants were introduced in 1941. The initial condition of these patients varied enormously, and their anticoagulant and antibiotic regime is often unspecified or clearly inadequate by modern standards, so a detailed analysis is unwarranted. However, two things are clear. Although there does not appear to be a report of a major disaster attributable to anticoagulants, there is indeed a risk of aggravating a haemorrhagic 
lesion. Thus, among the sixty-five patients treated with antibiotics alone, three were found to have haemorrhagic cerebral infarcts at autopsy, one died when a mycotic aneurysm ruptured, and two survived subarachnoid haemorrhages. Of the fortyseven patients treated with anticoagulants, seven had insignificant haemorrhages, or haemorrhages clearly attributable to gross overdosage, and one survived a period of coma and monoplegia with bloodstained CSF (Pirkey, 1950). Moreover, anticoagulant treatment failed to confer any definite benefit, for despite a slight reduction in the number of embolic episodes (14\% as against $23 \%$ ) the mortality rate was a little over $20 \%$ in each group. In view of the good prognosis of the mild case efficiently treated with antibiotics, and the greater risk of haemorrhage in severely ill patients with cerebral congestion, these figures suggest that the use of anticoagulants is unwarranted. It may be that in time fibrinolysins (Clune, 1963 ; Pascarelli \& Lemlich, 1964) or low molecular weight dextran (Leak \& Kemball Price, 1964) will prove to be safer and more effective.

\section{Prognosis}

Prior to the introduction of antibiotics this condition was nearly always fatal. Eighty per cent of patients now survive, but Shaw (1952), whose excellent review is quoted throughout this section, points out that only a quarter will escape serious complications or residual damage. Septicaemia, a CSF pleocytosis and involvement of both eyes are so common as to be part of the disease, and infection will spread to produce an orbital or cerebral abscess, meningitis or a subdural empyema (Kubik \& Adams, 1943) in over $25 \%$ of cases. These lesions, along with febrile fits in children and cerebral damage due to venous thrombosis or arterial emboli (Weisman, 1944) account for the frequent appearance of convulsions. Embolic episodes occur in $40 \%$ of cases, mainly in the lungs, but involvement of almost every part of the body has been reported. Subarachnoid haemorrhage has also been described (Eichhorn, 1941) on one occasion due to rupture of a mycotic aneurysm (Abraham et al., 1941).

It is hard to say how much permanent damage survivors will suffer, for slow improvement continues for a long time. One-third of the sixty patients reviewed by Shaw were left with clinical evidence of a third, fourth or sixth nerve palsy, and a quarter had a squint. Vision was impaired in nine, and lost unilaterally in five. Surprisingly, none was epileptic, and only three showed weakness of the limbs or a personality change-figures which suggest that those with severe cerebral damage rarely survive. One patient belatedly developed clinical evidence of pituitary damage (Williams, 1956) - a lesion which has been observed pathologically and suspected clinically during the acute stage (Weisman, 1944 ; Brown, 1961 ; Childress \& Bitzer, 1964).

\section{Thrombosis of the sagittal sinus and cortical veins Aetiology}

The variety of circumstances under which cerebral venous thrombosis may be encountered only becomes apparent when the aetiology of sagittal sinus and cortical venous thrombosis is considered. The problem is simplified if neoplasms, infections, trauma and haematological abnormalities are treated separately.

Occlusion of the sagittal sinus by meningiomas is well recognized, but the sinus may also be compressed by extradural metastases (Mones, 1965) and spontaneous intracerebral thrombosis has been described in association with carcinoma (Barnett \& Hyland, 1953). Most of the accounts of venous occlusion associated with infection are concerned with cavernous and lateral sinus thrombosis, but there are reports of cerebral venous thrombosis with meningitis (Bailey \& Hass, 1937), tonsillitis (Symonds, 1940), tuberculosis (Russell, 1944), typhoid (Martin \& Sheehan, 1941), diphtheria (Ford, 1960, p. 888) and mumps (Banker, 1961). This aspect of the problem deserves closer attention, for if cerebral venous thrombosis can occur as a sequel to pharyngeal infection and infections elsewhere, it may well be responsible for some of the cases of infantile hemiplegia following tonsillitis, tonsillectomy and minor infections in childhood, and for some cases of 'encephalitis' in adult life.

Among the earliest cases of post traumatic venous thrombosis described were those due to gunshot wounds in the head (Holmes \& Sargent, 1915). Closely allied to these is the thrombosis sometimes seen after surgery (Swanson \& Fincher, 1954) or the insertion of a needle into the sagittal sinus of a child (Ford, 1960, p. 888). However, trauma is not always so direct, and moulding of the head at birth, a fracture (depressed or otherwise) over the line of the sinus (Caudill, French \& Haines, 1953 ; Martin, 1955 ; Kinal, 1959 ; Beller, 1964) or even a blow on the head insufficient to produce unconsciousness or damage the skull may be followed by thrombosis of the sagittal or lateral sinus (Barnett \& Hyland, 1953). The thrombosis may form on post-traumatic haemorrhages in the wall of the sinus (Carrie \& Jaffé, 1953), because of injury to the dura or because of an extension of thrombosis in a diploic vein due in turn to trauma and infection to the skull and skin (Martin, 1955). Cerebral venous thrombosis may also occur spon- 
taneously as a result of the thrombotic diathesis that follows any operation or severe injury (Barnett \& Hyland, 1953) and it is tempting to suppose that some of the encephalitides and neurological disorders observed in patients with burns arise in this way (Emery \& Reid, 1962). In passing, it may be noted that venous congestion around a cortical venous thrombosis can be mistaken for a contrecoup injury (Barnett \& Hyland, 1953).

A liability to spontaneous thrombosis has been observed in patients with infections, severe injuries, cancer and a variety of other conditions. The thrombotic diathesis observed during the puerperium and, to a lesser extent, during pregnancy is the best known of these, but as the subject has been carefully reviewed recently it will not be considered again in this paper (Carroll, Leak \& Lee, 1966). Cerebral venous thrombosis may also occur in polycythaemia (Massachusetts General Hospital Clinicopathological Conference, 1960) in cyanotic congenital heart disease (Berthrong \& Sabiston, 1951) with right ventricular failure (Barnett \& Hyland, 1953) and in anaemic dehydrated infants (Bruno \& Ober, 1963). It has been encountered in sickle cell anaemia, especially during anaesthetics (Schenk, 1960) in diabetics (Ata, 1965), in homocystinuria (Dunn, Perry \& Dolman, 1966) and as an 'idiopathic' condition (Gerber \& Mendlowitz, 1949). It may also be responsible for the papilloedema sometimes seen in leukaemia (Barnett \& Hyland, 1953) and for some of the cerebrovascular accidents apparently precipitated by oral contraceptives (Lorentz, 1962).

\section{Clinical presentation}

The bewildering variety of clinical pictures presented by patients with thrombosis of the sagittal sinus and cortical veins is best understood if it is remembered that the gravity of the illness depends on the rate and extent of thrombosis, the potentialities of the collateral circulation, and the tendency for one syndrome to merge into the other. In general, sagittal sinus thrombosis produces the signs and symptoms of raised intracranial pressure, while cortical venous thrombosis causes fits, hemiplegia and depression of consciousness. One may therefore encounter a man with papilloedema who complained of headache some days or weeks after a minor head injury, a young mother who developed fits and a hemiplegia soon after delivery, or a dehydrated, rigid, comatose child with a bulging fontanelle, distended scalp veins, a crackpot note on percussion of the skull and gross papilloedema who was well apart from a little diarrhoea a few days before admission. But these patients and clinical pictures are to a large extent interchangeable, and no useful purpose is served by trying to analyse the general problem any further. It is instructive, however, to consider the unusual hemiplegia which results from thrombosis of the Rolandic vein.

The venous drainage of the cortex divides about a horizontal watershed running through the hand area, the sagittal sinus receiving blood which passes upwards from this line, and the cavernous sinus that which flows down. When the vein draining the Rolandic area thromboses, weakness develops slowly and intermittently, because collaterals compensate for the obstruction until they in turn are occluded. Loss of power is most severe and most persistent in the distal part of the lower limb, for the foot area is at the centre of the infarct. By contrast, any weakness that appears in the hand will be short-lived, because an alternative venous drainage is immediately available. The shoulder and hip areas, lying more deeply in the infarct, are more severely affected, but speech, sight and facial movement will be spared. Signs may be bilateral, as the sagittal sinus is often involved. The resulting picture of a conscious, coherent patient with full visual fields, normal facial movements, strong dextrous hands, a weak shoulder, severe bilateral foot drop, little spasticity and sensory loss of cortical type is strikingly unlike that produced by an arterial stroke, and can only be explained on the basis of venous occlusion (Merwarth, 1942).

\section{Investigations}

The first object of investigation is to exclude other possible causes for the clinical picture such as a meningioma, a subdural haematoma after head injury, eclampsia or a subarachnoid haemorrhage in the perinatal period, and meningitis in a comatose child. Angiography may show incidentally that the sagittal sinus is compressed by an extradural metastasis (Mones, 1965) or that the sinus fails to appear in late films after the arterial tree has been clearly outlined (Askenasy, Kosary $\&$ Braham, 1962). These findings, a fracture over the line of the sinus, or the discovery of erythrocytes in the CSF lend support to the diagnosis of thrombosis. Unfortunately, so little is known about electroencephalographic changes in this condition that the investigation is really only of value as a guide to progress (Lemmi \& Little, 1960). Incidentally, it must be remembered that the damaged hemisphere may become very swollen, so that the discovery of displaced vessels at angiography is compatible with a diagnosis of cortical venous thrombosis (Swanson \& Fincher, 1954).

\section{Treatment}

Treatment of the underlying disease rarely has any effect on the neurological picture, except in the 
case of removal of a meningioma, irradiation of a secondary deposit (Mones, 1965) or elevation of a depressed fracture (Caudill et al., 1953; Kinal, 1959). Few attempts have been made to use anticoagulants, and the frequent finding of erythrocytes in the CSF and cerebral haemorrhages at autopsy suggest that they have no place in the management of these lesions. The value of low molecular weight dextran has not yet been studied. Infection should, of course, be treated vigorously, and anticonvulsants will be required if fits or a hemiplegia suggest cortical damage. The management of intracranial hypertension will be considered in the section on lateral sinus thrombosis.

\section{The problem of diagnosis and prognosis}

This type of venous thrombosis is more frequently diagnosed at autopsy than during life, partly because the possibility is not even considered, and partly because the signs and symptoms are often too nondescript to allow a firm diagnosis to be made. Even at autopsy the lesion is liable to be missed if the patient survives the acute episode and the vessel recanalizes. Thrombosis of the cortical veins and sagittal sinus has therefore acquired an unduly evil reputation because it is almost always recognized when it causes a rapidly fatal illness, and is very often misdiagnosed when it produces isolated fits or an obscure encephalopathy, followed perhaps by dementia, a hemiplegia or involuntary movements (Dekaban \& Norman, 1958 ; Banker, 1961). For this reason, it is virtually impossible to comment on the prognosis in a satisfactory manner. In general, however, the prognosis for survival after cortical venous thrombosis is good, although many patients are hemiplegic or epileptic. Sagittal sinus thrombosis is a much greater threat to life, and the mortality during infancy and pregnancy is high. Post-traumatic thrombosis appears to be much less dangerous (Beller, 1964) although death has followed an injury too mild to fracture the skull or produce unconsciousness (Askenasy et al., 1962).

\section{Lateral sinus thrombosis}

Lateral sinus thrombosis is usually due to infection in the middle ear or pharynx, but may be associated with any of the conditions described above, and especially with mild occipital head injuries. Acute otitis media has been a relatively unimportant cause since the introduction of antibiotics, and at present the lesion is most likely to be encountered in patients with chronic or inadequately treated middle ear disease. In the absence of neurological signs, the diagnosis is suggested by rigors, persistent fever and headache, and tenderness over the jugular bulb.
Neurological complications usually appear between 3 days and 3 weeks after the onset of the infection, and include cranial nerve palsies, intracranial hypertension, cortical venous thrombosis and various inflammations. Apart from facial weakness due to the causative lesion, all are the result of extension of the thrombus. The sixth nerve is frequently damaged as it lies alongside an occluded and swollen inferior petrosal sinus in the fibrous loop known as Dorello's canal, the fifth nerve is occasionally compressed in the same way by the superior petrosal sinus, and the ninth, tenth and eleventh nerves may be damaged by the jugular bulb. Intracranial hypertension appears if the thrombus extends to occlude the torcula or if the opposite sinus is rudimentary. (Unlike a cerebral abscess, this condition rarely produces much in the way of symptoms apart from a little headache and vomiting, and the diagnosis is usually made when a patient with diplopia is found to have gross papilloedema.) Spread of the thrombus along veins draining the temporal lobe will produce cortical damage, with the risk of fits or a hemiplegia, and an infected thrombus may cause an extradural, subdural or cerebral abscess or meningitis as it traverses these territories (Symonds, 1940, 1956).

\section{Investigations}

In the acute stage, the main aims of investigation are the exclusion of other conditions which could be responsible for the clinical picture, such as a cerebral abscess or a subdural haematoma, and the early detection of visual failure. Study of the underlying disease is of subsidiary importance, and demonstration of the clot itself incidental.

The CSF pressure is often moderately increased, and thrombosis of the lateral sinus may be inferred if it fails to rise after jugular compression (the Tobey Ayer test). However, this is an unreliable and potentially fatal investigation (Symonds, 1952) and it is best avoided because angiography, ventriculography or exploration of the sinus will usually be required and will provide the same information. Changes in the composition of the CSF in cerebral venous thrombosis generally are so varied as to be almost without value for diagnostic purposes, but normal findings would be a point against the presence of a cerebral abscess (Bradshaw, 1956). The electroencephalogram may also help to exclude an abscess, but is of little value otherwise save as a supplement to clinical examination in assessing progress (Lemmi \& Little, 1960). Ventriculography shows that the ventricles are on the small side of normal, and there is no displacement of the cerebral vessels on angiography. However, careful study of the venous phase may show that the sinus fails to fill despite 
good filling of the arterial tree. A sinugram may demonstrate the clot still more accurately (Kinal \& Jaeger, 1960) but this is a relatively major undertaking which may cause further thrombosis or an air embolus, and is probably best avoided. In the presence of intracranial hypertension, careful charting of the visual fields, recording of visual acuity and fundus photographs are required so that visual failure can be detected at an early stage.

\section{Management}

Once the possibility of a cerebral abscess has been excluded, treatment depends mainly on the effective use of antibiotics and on surgical management of the causative lesion, often combined with ligation of the jugular vein and evacuation of the thrombus (Reading \& Schurr, 1956). If fits or a hemiplegia are observed, anticonvulsants should be given, but anticoagulants do not appear to have been used or to be needed. Indeed, the finding of haemorrhagic lesions in the brain at autopsy suggests that they may be dangerous (Bailey \& Hass, 1937). In the presence of papilloedema, an attempt should be made to reduce the venous pressure by raising the head and paying attention to the airway (Kinal, 1959). Lumbar punctures should be avoided, as they are potentially dangerous (Symonds, 1952) and there is little reason to believe that they lower pressure, speed recovery or reduce the slight risk of blindness. The value of diuretics and decompression is also questionable, but if there is a history of paroxysmal headache or episodic amblyopia, or clinical evidence of failing vision, surgical decompression is indicated (Bradshaw, 1956).

\section{Prognosis}

Lateral sinus thrombosis has become a rare disease, and with modern treatment the prognosis for life is excellent provided a cerebral abscess does not appear. The diagnosis was only made twelve times in Denmark between 1956 and 1960. Five of these patients recovered completely, but three of the four who developed a cerebral abscess died. Two survivors had slight facial weakness, one complained of tinnitus and vertigo, and one was epileptic (Jensen, 1962). Thromboembolic complications of varying severity (Reading \& Schurr, 1956) and weakness following cortical venous thrombosis will also be encountered occasionally. Patients with intracranial hypertension usually make a complete recovery and the risk of blindness is much less than with benign intracranial hypertension, although papilloedema may persist for months or years (Foley, 1955 ; Bradshaw, 1956).

\section{Thrombosis of the vein of Galen}

The full clinical picture of thrombosis of the vein of Galen is perhaps the most striking of all the syndromes under discussion. It is usually a sequel of severe deformity of the skull during a difficult premature or breech delivery, and it produces bilateral extrapyramidal signs with spastic weakness of both legs. These findings are only intelligible when it is appreciated that they are the result of cerebral softening in the area drained by the vein of Galen - the basal ganglia and the central white matter of both hemispheres (Benda, 1952). A similar neurological picture has been reported following a non-fatal illness in infancy (Banker, 1961) and in one such patient a suppression-burst electroencephalogram was recorded (Lemmi \& Little, 1960). But in infancy and adult life, thrombosis of the vein of Galen usually causes a rapidly fatal illness characterized by headache, mydriasis, hyperthermia, fits, coma and death (Garcin \& Pestel, 1949 ; Ford, 1960, pp. 887 and 889).

\section{Conclusion}

There is ample scope for further study of all aspects of this problem. Clinically, the diagnosis must be considered in every patient with an acute cerebral illness, especially in the presence of bilateral signs, weakness of unusual distribution or a cause for venous thrombosis. Radiologically, more attention must be paid to the venous phase of the angiogram, to detect abnormalities and to establish the range of normal findings. Therapeutically, observations on the value of low molecular weight dextrans are needed, especially in cortical venous thrombosis. Pathologically, the gross and microscopic appearance of the sinuses must be studied, for superficially a thrombosed sinus may look normal after recanalization. The clinical picture of cavernous and lateral sinus thrombosis is fairly easy to recognize, but this is not so with thrombosis of the cortical veins, sagittal sinus or vein of Galen. Only occasionally will classical signs, surgical observations or autopsy findings allow the diagnosis to be made unequivocally, but with careful study a few more obscure cases of hemiplegia, involuntary movements or encephalitis could be correctly diagnosed.

\section{Acknowledgment}

I would like to thank Dr P. C. Gautier Smith for his advice and help with the writing of this paper.

\section{References}

Abraham, E.P., Chain, E., Fletcher, C.M., Florey, H.W., Gardner, A.D., Heatley, N.G. \& Jennings, M.A. (1941) Further observations on penicillin. Lancet, ii, 177.

Askenasy, H.M., Kosary, I.Z. \& BrahaM, J. (1962) Thrombosis of the longitudinal sinus. Neurology (Minneap.), 12, 288. 
AtA, M. (1965) Cerebral infarction due to intracranial sinus thrombosis. J. clin. Path. 18, 636.

BaILEY, O.T. \& Hass, G.M. (1937) Dural sinus thrombosis in early life. J. Pediat. 11, 755.

BANKER, B.Q. (1961) Cerebral vascular disease in infancy and childhood. J. Neuropath. exp. Neurol. 20, 127.

BarNetT, H.J.M. \& Hyland, H.H. (1953) Non infective intracranial venous thrombosis. Brain, 76, 36.

Barraquer Bordas, L., Bachs, A. \& Barraquer-Moner, J. (1955) La forme subaigue de la thrombophlébite de sinus caverneux. Presse med. 63, 376.

Beller, A.J. (1964) Benign post traumatic intracranial hypertension. J. Neurol. Neurosurg. Psychiat. 27, 149.

BENDA, C.E. (1952) Developmental Disorders of Mentation and Cerebral Palsies, p. 259. Grune \& Stratton, New York.

Berthrong, M. \& Sabiston, D.C. (1951) Cerebral lesions in congenital heart disease. Bull. Johns Hopk. Hosp. 89, 384.

BRADSHAW, P. (1956) Benign intracranial hypertension. $J$. Neurol. Neurosurg. Psychiat. 19, 28.

Brown, P. (1961) Septic cavernous sinus thrombosis. Bull. Johns Hopk. Hosp. 109, 68.

BrUNo, M.S. \& Ober, W.B. (1963) Diarrhoea, vomiting, fever and seizures in an infant. N.Y. St.J. Med. 63, 841.

Carrie, A.W. \& JafFe, F.A. (1953) Thrombosis of superior sagittal sinus caused by trauma without penetrating injury. J. Neurosurg. 11, 173.

Carroll, J.D., Leak, D. \& Lee, H.A. (1966) Cerebral thrombophlebitis in pregnancy and the puerperium. Quart. J. Med. 35, 347.

Caudill, C.M., French, L.A. \& Haines, G.L. (1953) Increased intracranial pressure following compression of the superior sagittal sinus. Neurology (Minneap.), 3, 231.

Childress, R.C. \& Bitzer, W. (1964) Cavernous sinus thrombosis. J. Fla med. Ass. 51, 94.

Clune, J.P. (1963) Septic thrombosis within the cavernous chamber. Amer. J. Ophthal. 56, 33.

DeKaban, A.S. \& Norman, R.M. (1958) Hemiplegia in early life associated with thrombosis of the sagittal sinus and its tributary veins in one hemisphere. J. Neuropath. exp. Neurol. 17, 461.

Dunn, H.G., Perry, T.L. \& Dolman, C.L. (1966) Homocystinuria. A recently discovered cause of mental defect and cerebrovascular thrombosis. Neurology (Minneap.), 66, 407.

EICHHORN, J.P. (1941) Cavernous sinus thrombosis. Ohio St. med. J. 37, 1076.

EmerY, J.L. \& ReID, D.A.C. (1962) Cerebral oedema and spastic hemiplegia following minor burns in young children. Brit. J. Surg. 50, 53.

FolEY, J. (1955) Benign forms of intracranial hypertension'toxic' and 'otitic' hydrocephalus. Brain, 78, 1.

FORD, F.R. (1960) Diseases of the Nervous System in Infancy, Childhood and Adolescence, 4th edn, pp. 887-889. Blackwell Scientific Publications, Oxford.

Garcin, R. \& Pestel, M. (1949) Thrombophlebites Cerebrales, p. 107. Masson, Paris.

Gerber, I.E. \& Mendlowitz, M. (1949) Visceral thrombophlebitis migrans. Ann. intern. Med. 30, 560.

Holmes, G. \& SARgent, P. (1915) Injuries of the superior longitudinal sinus. Brit. med. J. ii, 493.
Jensen, A.M. (1962) Sinus thrombosis and otogene sepsis Acta otolaryng. (Stockh.), 55, 237.

KINAL, M.E. (1959) Traumatic thrombosis of dural venous sinuses. First International Congress of Neurological Sciences (Ed. by L. Van Bogaert and J. Radermecker), vol. 2, p. 178. Pergamon Press, London.

Kinal, M.E. \& JAEGER, R.M. (1960) Thrombophlebitis of dural venous sinuses following otitis media. J. Neurosurg. $17,81$.

KUBIK, C.S. \& AdAMS, R.D. (1943) Subdural empyema. Brain, 66, 18.

Leak, D. \& Kemball Price, R. (1964) Low molecular weight dextran in cerebral ischaemic episodes. Lancet, $\mathbf{i}, 772$.

LemMI, H. \& LitTLE, S.C. (1960) Occlusion of intracranial venous structures. Arch. Neurol. (Chic.), 3, 252.

LORENTZ, I.T. (1962) Parietal lesion and 'enavid'. Brit. med. J. ii, 1191.

MARTIN, J.P. (1955) Signs of obstruction of the superior longitudinal sinus following closed head injuries. Brit. med. J. ii, 467.

Martin, J.P. \& Sheehan, H.L. (1941) Primary thrombosis of cerebral veins (following childbirth). Brit. med. J. i, 348.

Massachusetts General Hospital (1960) Case Report No. 46471. New Engl. J. Med. 263, 1080.

MERWARTH, H.R. (1942) The syndrome of the Rolandic vein. Amer. J. Surg. 56, 526.

MONES, R.J. (1965) Increased intracranial pressure due to metastatic disease of venous sinuses. Neurology (Minneap.), 15, 1000.

Pascarelli, E. \& Lemlich, A. (1964) Diplopia and photophobia as premonitory symptoms in cavernous sinus thrombosis. Ann. Otol. (St. Louis), 73, 210.

PIRKEY, W.P. (1950) Thrombosis of the cavernous sinus Arch. Otolaryng. 57, 917.

Reading, P.V. \& SchurR, P.H. (1956) Thrombosis of the sigmoid sinus. Lancet, ii, 473.

Russell, D.S. (1944) Venous thrombosis in the central nervous system. Proc. roy. Soc. Med. 37, 391.

SCHENK, E.A. (1960) Sickle cell trait and superior longitudinal sinus thrombosis. Ann. intern. Med. 60, 465 .

SHAw, R.E. (1952) Cavernous sinus thrombosis: a review. Brit. J. Surg. 40, 40.

Swanson, H.S. \& Fincher, E.F. (1954) Experiences involving the superior longitudinal sinus and Rolandic veins. Neurology (Minneap.), 4, 801.

Symonds, C.P. (1940) Cerebral thrombophlebitis. Brit. med. J. ii, 348.

SymoNDs, C. (1952) Intracranial thrombophlebitis. Ann. roy. Coll. Surg. Engl. 10, 347.

Symonds, C. (1956) Otitic hydrocephalus. Neurology (Minneap.), 10, 681.

TAYLOR, P.J. (1957) Cavernous sinus thrombophlebitis. Brit. J. Ophthal. 41, 228.

Weisman, A.D. (1944) Cavernous sinus thrombophlebitis. New Engl. J. Med. 231, 118.

Williams, E. (1956) Hypopituitarism following sinusitis and cavernous sinus thrombosis. Proc. roy. Soc. Med. 49, 827. 